\title{
Urinary Neutrophil Gelatinase - Associated Lipocalin as an Early Marker of Diabetic Nephropathy in Children and Adolescents
}

\author{
Shereen Abdelghaffar Taha ${ }^{(1)}$, Ashraf Sayed Kamel ${ }^{(2)}$, Hoiyda Ahmed Abdelrasul ${ }^{(3)}$ \\ ,Mostafa Rabie Ahmed Abdelbaki ${ }^{(4)}$ \\ (1)Professor of Pediatrics,Faculty of Medicine, Cairo University \\ (2)Assistant Professor of Pediatrics,Faculty of Medicine, Fayoum University \\ (3)Professor of Clinical Pathology,Faculty of Medicine, Fayoum university \\ (4) (M.B.B.Ch).
}

Corresponding Author: prof. Ashraf Sayed Kamel

Mail:Ashraf5kam@yahoo.com

Tel:01225876153

\begin{abstract}
Diabetic nephropathy is one of the most important complications of type 1 diabetes in children and adolescents.

Our aim of the study is to determine whether urinary neutrophil gelatinase- Associated lipocalin (NGAL) levels can be considered as an early marker of Diabetic kidney injury.

This study was a case control study which included 84 Children who were following up at Diabetes, Endocrinology and metabolism pediatric clinic in Abo- Elreesh university hospital and general pediatric clinic in Fayoum university hospitals. Participants were divided into three groups. Group 1 included 29 patients with diabetic nephropathy (microalbuminuria), Group 2 included 27 patients with type 1 diabetes mellitus without nephropathy (normoalbuminuria) and group 3 included 28 healthy controls who were age and sex matched with diabetic Children.

Our study showed that Mean value of Urinary NGAL was higher in microalbumiuric group (mean \pm SD $164.3 \pm 21.5 \mathrm{ng} / \mathrm{ml}$ ) than in normoalbuminuric group (mean \pm SD $127.27 \pm 17.58 \mathrm{ng} / \mathrm{ml}$ ) and the control group $($ mean \pm SD $103.91 \pm 33.88 \mathrm{ng} / \mathrm{ml})$ with $\mathrm{p}$-value $=\mathbf{< 0 . 0 0 0 1}$.

We found that urinary NGAL measurement might become an early, sensitive, practical and noninvasive accurate tool for early detection of diabetic kidney injury in children and adolescents with type 1 diabetes mellitus and even before development of microalbuminuria.
\end{abstract}

\section{KEYWORDS: Diabetic nephropathy, neutrophil gelatinase - associated lipocalin, Microalbuminuria, Normoalbuminuria}

\section{INTRODUCTION}

Diabetes mellitus, the most common endocrine disorder of childhood, is a common group of metabolic disorders that share the phenotype of hyperglycemia resulting from diminished and /or ineffective insulin action [1].

The metabolic dyregulation with diabetes results in pathophysiologic changes in multiple organs which results in different complications which needs continuous medical care and ongoing patient selfmanagement, education and support to prevent morbidities and mortality from these complications [2].

Diabetic nephropathy (DN) is one of the most important complications of type 1 diabetes which causes many morbidity and mortality cases in children and adolescents [3].

Microalbuminuria emerged as a marker of renal damage in diabetic nephropathy; however, this measurement lacks the sensitivity to detect the earliest changes in renal function as it has been known that some histopathological changes related to DN emerge before the onset of micro -albuminuria [4]. 
The approach to Diabetic nephropathy in diabetic patients is to initiate the renin-angiotensin-system (RAS) inhibition once micro albuminuria. Thus the early recognition of diabetic kidney injury before the microalbuminuric phase is essential to prevent end stage renal disease (ESRD) related to DN [5].

Noninvasive methods to diagnose DN in earlier stages have been investigated in some studies. These studies mostly focused on neutrophil gelatinase-associated Lipocalin (NGAL) which is the most promising biomarker for early detection of acute kidney injury [6].

Neutrophil gelatinase - associated lipocalin (NGAL), a member of the lipocalin protein superfamily is released from the renal tubular cells in response to various acute and chronic insults to the kidney such as diabetic nephropathy, contrast nephropathy, $\operatorname{IgA}$ nephropathy and lupus nephritis [7].In contrast to conventional markers, such as serum creatinine, blood urea nitrogen, or serum cystatin C (CysC), NGAL is not considered a marker of renal function, but rather reflects structural damage of renal cells [7].

Higher urinary levels of NGAL have been found in diabetics compared to controls, even in normoalbuminuric patients, indicating that diabetic tubular damage may develop before the stage of micro albuminuria. The urinary NGAL level already was significantly elevated in normoalbuminuric patients with diabetes compared with non-diabetic controls and was significantly associated with albuminuria. Mean urinary NGAL and NGAL/creatinine ratio $(\mathrm{NGAL} / \mathrm{Cr})$ levels in both microalbuminuric and nonmicroalbuminuric diabetic patients were found to be higher than those in the controls, indicating that tubular involvement may precede glomerular involvement [8].

As urinary NGAL levels are increased in the very early phase of diabetes before micro albuminuria develops, the increased levels of urinary NGAL in diabetic patients, with or without albuminuria, point to early tubular damage and can be used as an early sensitive marker in detecting diabetic kidney disease (DKD) [9]. Furthermore, the combination of novel therapies and well-established conventional treatments might reduce the tremendous burden associated with diabetic nephropathy [9].

\section{PATIENTS AND METHODS}

\section{Study design}

This study was a case control study.

\section{Subjects}

The study included 84 Children who were following up at Diabetes, Endocrinology and metabolism pediatric clinic in Abo- Elreesh university hospital and general pediatric clinic in Fayoum university hospitals. Participants were divided into three groups. Group 1 included 29 patients with diabetic nephropathy (microalbuminuria), Group 2 included 27 patients with type 1 diabetes mellitus with normoalbuminuria and group 3 included 28 healthy controls who were age and sex matched with diabetic Children and with no other diseases.

\section{Patients were recruited according to the following criteria}

\section{Inclusion criteria}

Children diagnosed with type 1 diabetes mellitus 2-18 years old According to guidelines of ISPAD 2014

Duration of diabetes more than 2 years

Both genders

\section{Exclusion criteria}

1. Patients with metabolic disorders, degenerative or autoimmune diseases other than diabetes mellitus 
Duration of diabetes less than 2 years

Age $<2$ years or above 18 years

Children with end stage renal disease

\section{All cases were subjected to the following}

\section{History taking in the form of}

- Age and sex of the patients

- Duration of diabetes in years

- Age of onset of diabetes

- Family history of diabetes

- Family history of kidney diseases

- Insulin therapy regimen

- The daily total dose of insulin and the type of insulin

- History and frequency of acute metabolic complications:

1-History suggestive of DKA (any attacks of DKA at diagnosis or during follow up).

2-History suggestive of hypoglycemic attacks (tremors, convulsions or coma).

- History suggestive of chronic Diabetic complications

1-Peripheral neuropathy manifestations (tingling, numbness and parathesia).

2-Diabetic nephropathy

- Previous hospital admission

- History of previous viral illness

\section{Clinical examination with special emphasis on}

- Anthropometric measurements including

- Standing height and height standard deviation score (SDS) for age and Sex

- Weight and weight SDS for age and sex

- Body mass index (BMI) and BMI standard deviation score

- Blood pressure measured by the conventional mercury sphygmo-manometer in the laying position then plotted on blood pressure curves according to the age and sex

\section{Laboratory investigations}

A. Spot urinary albumin creatinine ratio. (Patient has persistent micro -albuminuria according to albumincreatinine ratio (A/C) calculated in morning urine sample. An A/C ratio 30-300 $\mu \mathrm{g} / \mathrm{mg}$ is considered positive for microabuminuria in absence of urinary tract infection. Two positive results for microalbuminuria within 3 months should be done.

B. Follow up records (HbA1C) last year.

C. Serum creatinine and calculating the glomerular filtration rate (GFR) from the Schwartz formula

$$
\mathrm{GFR}=\underline{\mathrm{K} \times \text { height }(\mathrm{cm})}
$$

\section{S.creatinine $(\mathrm{mg} / \mathrm{dl})$.}

$\mathrm{K}$ is constant calculated as follow

Where $\mathrm{k}$ is 0.55 for children and adolescent females, and 0.70 for adolescent males

D. Urinary neutrophil gelatinase- associated lipocalin measurement using ELISA technique.

E. Mean fasting blood glucose

F. Mean postprandial blood glucose

G. Lipid profile ( cholesterol,triglycerides,HDL,LDL) 


\section{Collection of samples and storage}

First morning urine specimens were obtained to measure uNGAL. Immediately after being collected, Centrifuged at (2000-3000 RPM) for approximately 20 minutes. The supernatants collected carefully and the samples stored at $-20{ }^{\circ} \mathrm{C}$ and analyzed all at the same time.

\section{Principle of assay of uNGAL}

This assay done using uses enzyme-linked immune sorbent assay (ELISA) based on the Biotin double antibody sandwich technology to assess the Human Neutrophil gelatinase associated lipocalin (NGAL). Neutrophil gelatinase-associated lipocalin (NGAL) added to the wells, which are pre-coated with Neutrophil gelatinase-associated lipocalin (NGAL) monoclonal antibody and then incubated. After that, anti NGAL antibodies labeled with biotin added to unite with streptavidin-HRP, which forms immune complex. Unbound enzymes removed after incubation and washing. Substrate A and B added. Then the solution will turn blue and change into yellow with the effect of acid. The shades of solution and the concentration of Human Neutrophil gelatinase-associated lipocalin (NGAL) are positively correlated.

\section{RESULTS}

\begin{tabular}{|c|c|c|c|c|c|c|c|}
\hline & \multicolumn{2}{|c|}{$\begin{array}{c}\text { Micro- } \\
\text { albuminuria } \\
(\mathrm{N}=29)\end{array}$} & \multicolumn{2}{|c|}{$\begin{array}{c}\text { Normoalbuminuria } \\
(\mathrm{N}=27)\end{array}$} & \multicolumn{2}{|c|}{$\begin{array}{c}\text { Controls } \\
(\mathrm{N}=28)\end{array}$} & P-value \\
\cline { 2 - 8 } & $\begin{array}{c}\text { Mean } \\
\text { SD }\end{array}$ & Mean & SD & Mean & SD & \\
\hline $\begin{array}{c}\text { Urinary NGAL } \\
(\mathrm{ng} / \mathrm{mL})\end{array}$ & 164.3 & 21.5 & 127.27 & 17.58 & 103.91 & 33.88 & $<\mathbf{0 . 0 0 0 1 *}$ \\
\hline
\end{tabular}

NGAL $=$ urinary neutrophil gelatinaseassociated lipocalin
This study shows that there was statistically significant difference among study groups as regard Urinary NGAL levels. Mean value of Urinary NGAL was higher in microalbumiuric group than in normoalbuminuric and the control group

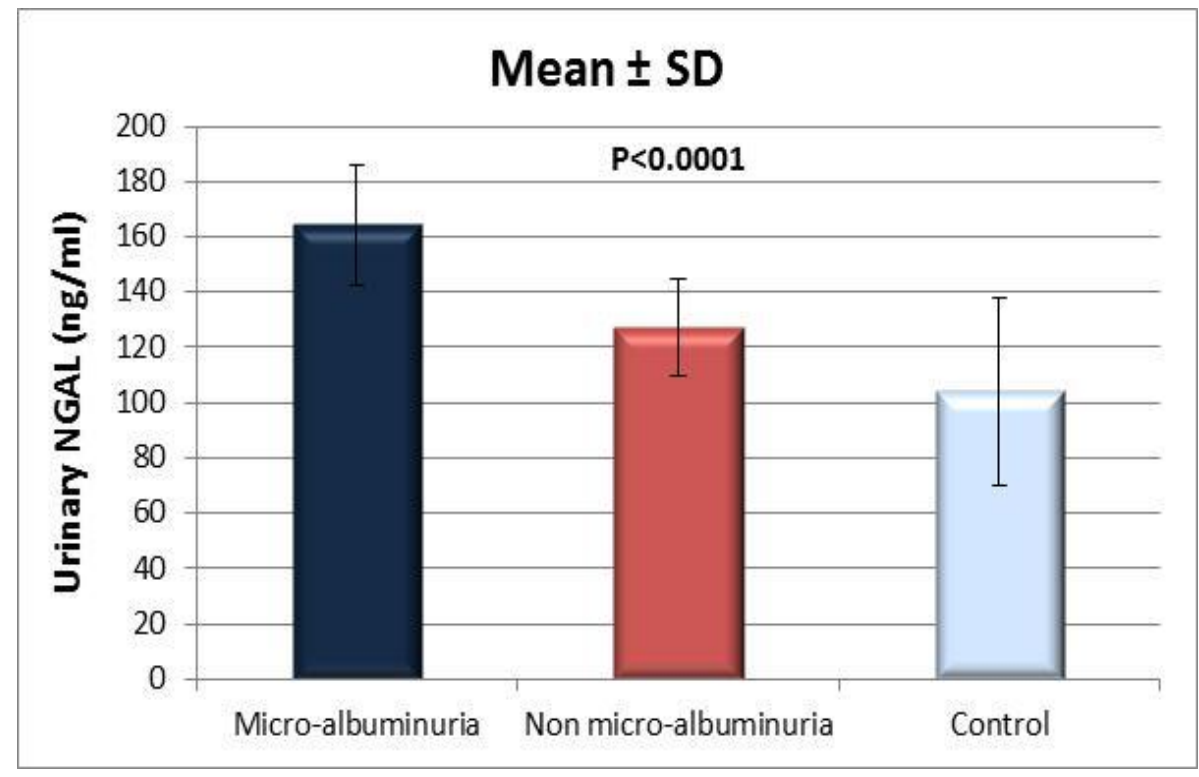


Receiver operator characteristic (ROC) curves NGAL in detecting microalbuminuria in diabetic Showed better diagnostic accuracy of urinary patients.

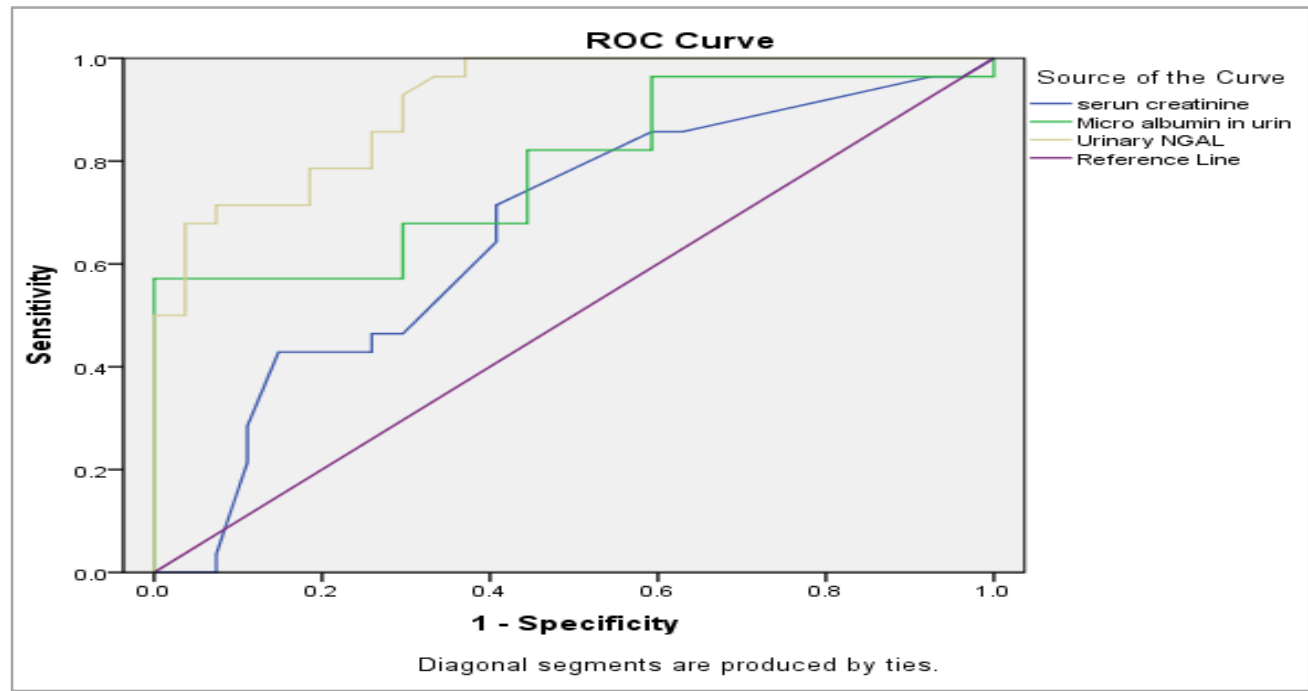

Receiver operator characteristic (ROC) curves Showed The AUC was significantly higher for uNGAL with Pbetter diagnostic accuracy of urinary NGAL than value $<\mathbf{0 . 0 0 0 1}$, but not for serum creatinine with Pserum creatinine in detecting microalbuminuria in ${ }^{\text {value }}=\mathbf{0 . 0 3 8}$ and microalbumin in urine with $p$ diabetic patients. The sensitivity and specificity of

serum creatinne, microalbumin in urine and uNGALThe sensitivity and specificity of uNGAL were $100 \%$ were estimated via ROC curves. The area under theand 63\%, respectively with the upper reference limit as curves (AUC) was $\mathbf{0 . 9 1 6}$ for uNGAL, $\mathbf{0 . 6 6 3}$ for serumthe cut-off.

creatinine and $\mathbf{0 . 7 7 7}$ for microalbumin in urine.

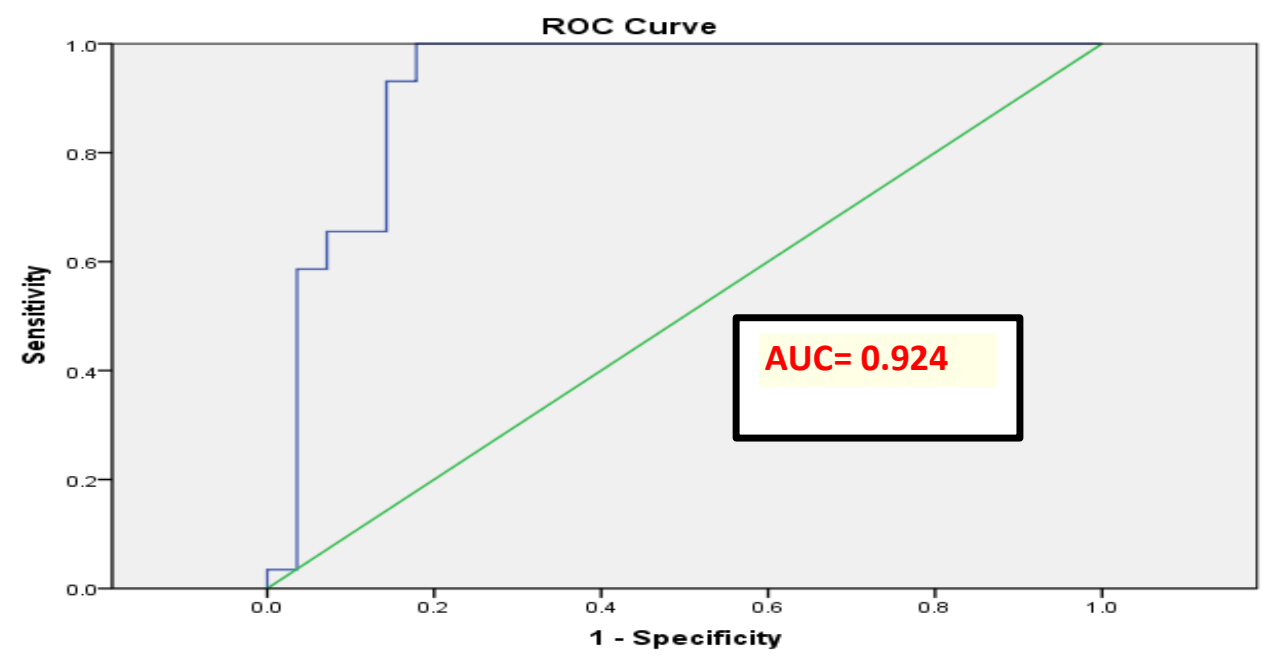

The above figure shows that in the microalbuminuric vs. control group AUC was 0.924, sensitivity $100 \%$ and specificity 82.1 at cut- off point 130.67 . 


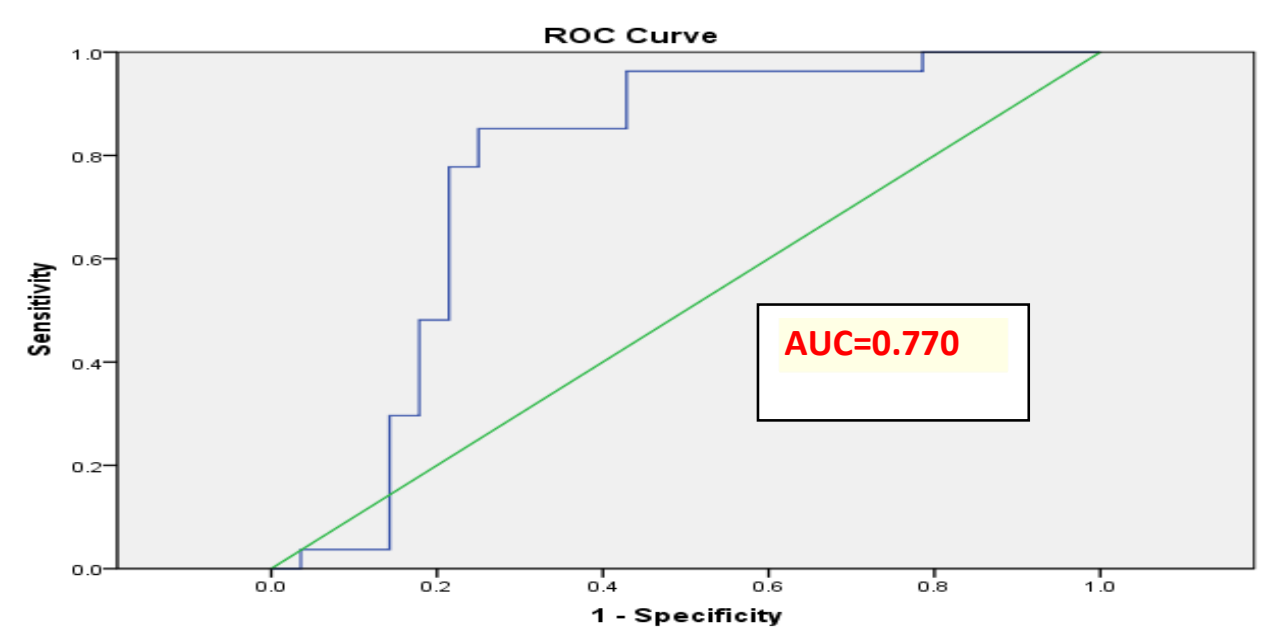

The above figure shows that in normoalbuminuric group vs. control group AUC is 0.770 with sensitivity $85.2 \%$ and specificity $75 \%$ at cut off point 114.63 .

\section{Comparison of sensitivity and specificity of urinary NGAL among study groups:}

\begin{tabular}{|c|c|c|c|c|c|}
\hline & AUC & P-value & $\begin{array}{c}\text { Cut-off } \\
\text { point }\end{array}$ & Sensitivity & Specificity \\
\hline $\begin{array}{c}\text { Micro-albuminuria vs. } \\
\text { Normoalbuminuria }\end{array}$ & 0.916 & $<\mathbf{0 . 0 0 0 1 *}$ & 132.57 & $100 \%$ & $63 \%$ \\
\hline $\begin{array}{c}\text { Micro-albuminuria vs. } \\
\text { control }\end{array}$ & 0.924 & $<\mathbf{0 . 0 0 0 1 *}$ & 130.67 & $100 \%$ & $82.1 \%$ \\
\hline $\begin{array}{c}\text { Normoalbuminuria vs. } \\
\text { control }\end{array}$ & 0.770 & $\mathbf{0 . 0 0 1 *}$ & 114.63 & $85.2 \%$ & $75 \%$ \\
\hline
\end{tabular}

\section{DISCUSSION}

Our study showed that mean value of Urinary NGALOur study revealed statistically significant positive was higher in microalbumiuric group (mean 164.3 teorrelation between A/C ratio and duration of DM this $21.5 \mathrm{ng} / \mathrm{ml} \mathrm{SD}$ ) than in normoalbuminuric groupmeans that the longer the duration of diabetes the (mean $127.27 \pm 17.58 \mathrm{ng} / \mathrm{ml} \mathrm{SD}$ ) and the control grouphigher the level of AC ratio.

(mean 103.91 $33.88 \mathrm{ng} / \mathrm{ml} \mathrm{SD}$ ). This attractive $\mathrm{We}$ also found that there was a significant positive finding supports the growing hypothesis of a "tubular correlation between A/C ratio and systolic and phase" of diabetic disease that precedes the diastolic blood pressure HbA1c, serum creatinine and manifestation of typical glomerular lesions. Thus, the micro albumin in urine.

increase in urine NGAL values may express the degree

The study demonstrated that after controlling of subclinical tubular impairment, representing an duration of diabetes, diastolic blood pressure, HBA1C earlier measurable index of renal distress compared and serum creatinine, systolic blood pressure and with classic glomerular signs. micro albumin in urine were statistically significant The value of urinary NGAL was found to be directlypositive predictors for AC ratio. proportional to the A/C ratio value, which mean that In our study, for Differentiating microthe more severe the kidney affection, the higher thealbuminuria from non-micro-albuminuria the area value of urinary NGAL. under the curve (AUC) was 0.916 of urinary NGAL The current study showed that after controlling age, with $100 \%$ sensitivity and specificity $63 \%$ at cut off height, duration of diabetes, systolic blood pressure point $132.57 \mathrm{ng} / \mathrm{mL}$. In the microalbuminuric vs. and HBA1C. AC ratio was found to be statisticallycontrol group $\mathrm{AUC}=0.924$ sensitivity $100 \%$ and significant predictor of urinary NGAL $(p<0.001)$. 
While in normoalbuminuric group vs. control group $75 \%$ at cut off point $114.63 \mathrm{ng} / \mathrm{mL}$. AUC $=0.770$ with sensitivity $85.2 \%$ and specificity

\section{CONCLUSION}

The present study has clearly demonstrated that High before microalbuminuria develops, and this in turn urinary levels of NGAL have been found in diabetic point to early tubular damage. So, urinary NGAL children compared to controls, and also levels were measurement might become an early, useful, sensitive, found to be higher in diabetic patients withpractical and noninvasive accurate tool for early microalbuminuria than in those with detection of diabetic kidney injury in children and

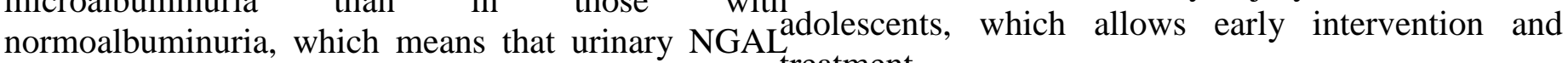
levels are increased in the early phase of diabetes treatment.

\section{REFERENCES}

[1] American Diabetes Association. Medical Management of Type 1 Diabetes. 7th Ed. Diabetes Care .2017; 41(Suppl. 1):S1-S2.

[2] Papadopoulou-Marketou N, Chrousos GP, Kanaka-Gantenbein C. Diabetic nephropathy in type 1 diabetes: a review of early natural history, pathogenesis, and diagnosis. Diabetes/metabolism research and reviews. 2017; 33(2).

[3] Kato M, Natarajan R. Diabetic nephropathyemerging epigenetic mechanisms. Nature Reviews Nephrology. 2014; 10:517-530

[4] Schmidt-Ott KM, Mori K, Li JY, Kalandadze A, Cohen DJ, Devarajan P, Barasch J: Dual action of neutrophil gelatinase-associated lipocalin. Journal of American Nephrology. 2007; 18(2):407.

[5] Haase-Fielitz A, Haase M, Devarajan P.

Neutrophil gelatinase-associated lipocalin as a biomarker of acute kidney injury: a critical evaluation of current status. Clinical Biochemistry .2014; 51 : 335-351.

[6] Abd EI Dayem S.A, E. El Bohy, and A. El Shehaby .Value of the intrarenal arterial resistivity indices and different renal biomarkers for early identification of diabetic nephropathy. Journal 2016; 29(3):273-9.

[7] Nielsen, Stine \& Schjoedt, Katrine \& S Astrup, A \& Tarnow, Lise \& Lajer, Maria \& Hansen, Peter \& Parving, H-H \& Rossing, Peter.Neutrophil Gelatinase-Associated Lipocalin (NGAL) and Kidney Injury Molecule 1 (KIM1) in patients with diabetic nephropathy: a cross-sectional study and the effects of lisinopril. Journal of the British Diabetic Association. 2010; 27(10):1144-50.

[8] Hafez MH, Fatma AF El-Mougy, Samuel H Makar, Semon S Abd El Shaheed.

Detection of an Earlier Tubulopathy in Diabetic
Nephropathy among Children with

Normoalbuminuria. Iranian Journal of Kidney Diseases; 2015, 9 (2):126-131.

[9] Zeynep Yuruk Yıldırım1, Ahmet Nayır1, ev Yılmaz1, Asuman Gedikbaşı2, Ruveyde Bundak.Neutrophil Gelatinase-Associated Lipocalin as an Early Sign of Diabetic Kidney Injury in Children. Journal of Clinical Pediatric Endocrinology. 2015; 7(4):274-279. 